\section{Intestinal gluconeogenesis prevents hepatic steatosis}

Intestinal gluconeogenesis (IGN), in which glucose produced in the small intestine is released into the portal vein, has shown protective effects against diabetes and obesity by positively regulating glucose homeostasis and hepatic glucose production. These effects have now been investigated in mice independently of nutritional manipulation and show that IGN has beneficial effects on hepatic steatosis and the initiation of nonalcoholic fatty liver disease (NAFLD).

"We previously demonstrated that IGN, via the sensing of glucose by the gastrointestinal neural system, signals to brain regions regulating energy homeostasis," explains lead author Gilles Mithieux. "Given the likely effect of IGN on the liver via brain-sympathetic-parasympathetic signalling, we considered the hypothesis of a beneficial action of IGN on hepatic steatosis and initiation of NAFLD."
The effects of IGN were previously demonstrated in mice in response to protein-enriched or fibre-enriched diets. "To avoid possible interference with effects of inducing nutrients that could be independent of IGN, we created a mouse model of activated IGN through overexpression of intestinal glucose 6-phosphatase (G6Pase), the key enzyme of gluconeogenesis, which was compared to wild-type mice and mice with intestine-specific knockout of G6Pase," explains Mithieux.

The researchers found that mice on a hypercaloric diet and overexpressing IGN in the gut were protected against development of hepatic steatosis. "The protection related to a diminution of de novo lipogenesis gene expression and lipid import, which was associated with decreased liver inflammation and fibrosis and the development of hepatic sympathetic nerves," reports
PANCREATIC CANCER

\section{Inducing senescence sensitizes pancreatic tumours to therapies}

Pancreatic ductal adenocarcinoma (PDAC) is typically characterized by a hypovascular, immunosuppressive tumour microenvironment and is often refractory to chemotherapies and immunotherapies. Now, a new study shows that senescence induction in mouse models of Kras-mutant PDAC sensitizes tumours to such therapies.

In the study, in two mouse models of PDAC, 2-week treatment with trametinib and palbociclib (T/P) - which inhibit

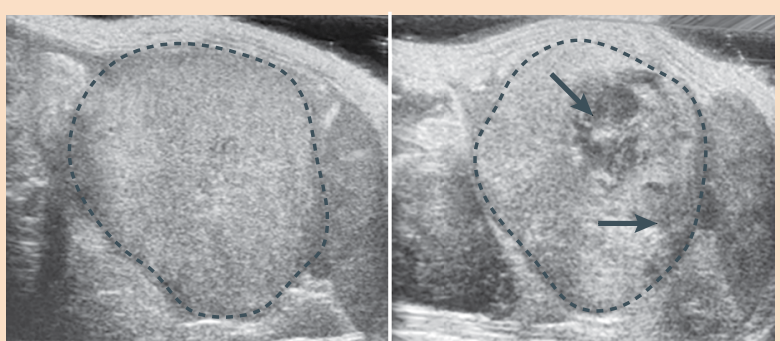

Credit: Ultrasound images from mice with PDAC organoids prior to (left) and after (right) treatment with T/P and a PD1 antibody. Dotted outlines indicate tumours, and arrows point to areas of tumour necrosis. Images courtesy of S.W. Lowe, M. Ruscetti, J.P. Morris IV and R. Mezzadra, Memorial Sloan Kettering Cancer Center, USA.
MEK and CDK4/6, respectively - led to induction of senescence-associated $\beta$-galactosidase activity in tumours and to decreased proliferation but not tumour regression. Intriguingly, T/P-treated tumours had improved vascularization.

Next, mouse and human PDAC cell lines as well as in vivo mouse models were used to show that the vascular remodelling is driven by senescenceassociated secretory phenotype factors. These factors, which included vascular endothelial growth factor and various inflammatory molecules, altered endothelial cell proliferation and endothelial activation, respectively. "The impact of senescence on the PDAC vasculature was completely unexpected," says Scott Lowe, corresponding author of the study.

Finally, the researchers investigated the effects of senescence induction on the efficacy of chemotherapies and immunotherapies in mouse models of PDAC. Gemcitabine (standard-of-care
Credit: Haematoxylin and eosin staining of mouse liver tissue from wild-type mice (above) and mice with overexpression of intestinal glucose 6-phosphatase (below). Images courtesy of G. Mithieux, Institut national de la santé et de la recherche médicale, France.

Mithieux. Conversely, IGN-deficient mice on a standard starch-enriched diet exhibited increased hepatic triglyceride storage and lipogenesis, which was corrected by a portal glucose infusion mimicking IGN.

"Our data are proof of the concept that activation of G6Pase in the gut is sufficient to activate IGN and prevent serious obesity-associated complications, such as NALFD and diabetes," concludes Mithieux. "This paves the way for future approaches of prevention or treatment of metabolic diseases, starting with the search of potential activators of G6Pase restricted to the intestine." The team also plan to further investigate the mechanisms of the effects induced by IGN, such as the prevention of body weight gain.

Iain Dickson

ORIGINAL ARTICLE Vily-Petit, J. et al. Intestinal gluconeogenesis prevents obesity-linked liver steatosis and non-alcoholic fatty liver disease. Gut https://doi.org/10.1136/gutjnl-2019-319745(2020) chemotherapy) combined with T/P increased treatment delivery to the tumour and led to large tumour regressions and significantly enhanced survival compared with mice treated with either T/P or gemcitabine alone. In addition, T/P-treated tumours had a substantial influx of PD1-expressing $\mathrm{CD}^{+} \mathrm{T}$ cells into the tumour microenvironment. Mice treated with T/P and anti-PD1 therapy had tumour regressions and a greater than fivefold increase in survival compared with mice treated with PD1 alone.

"Our findings suggest that senescenceinduced vascular remodelling could be a powerful way to potentiate both chemotherapy and immunotherapy in solid tumours that have a dysfunctional blood vessel network, such as pancreatic cancer," notes Lowe. "We are currently in the process of establishing a clinical trial to test the combination of MAPK and CDK4/6 inhibitors with PD1 blockade in patients with pancreatic cancer."

Jordan Hindson

ORIGINAL ARTICLE Ruscetti, M. et al.

Senescence-induced vascular remodeling creates therapeutic vulnerabilities in pancreas cancer. Cell 181, 424-441.e21 (2020) 